\title{
Application of Core Strength Training in Badminton Sport
}

\author{
Jikang Wang \\ Department of Physical Education \\ Shaanxi Xueqian Normal University \\ Shaanxi, China 710100
}

\author{
Juan Cheng \\ Department of Physical Education \\ Shaanxi Xueqian Normal University \\ Shaanxi, China 710100
}

\begin{abstract}
Badminton is a kind of national sport which can bring other parts of the body movement and need highly coordination of the power of feet, body and arms in order to improve the quality of the shuttle. The core strength emphasizes on stabilizing the core parts of players through controlling the power of arms and feet to reach the control of shuttle on the formation and trajectory of movement. Therefore, the impact of the core strength in the movement of badminton players should not be overlooked. So, there are a lot of competitive events focusing on the core strength training and it has gradually been scheduled on the sports training and research. This article analyzes the definition and characteristics of core strength as well as the important role of the core strength in badminton, which explores how to strengthen the training of the core strength of badminton players, aiming to providing reference for badminton in the core strength training. The article analyzes the definition and characteristics of core strength and analyzes the important role of the core strength in the badminton sport through the method of data discourse so as to explore how to strengthen the training of the core strength of badminton players in order to provide the badminton fans with the core strength training for reference.
\end{abstract}

Keywords-shuttle; the core strength; the core strength training; application

\section{INTRODUCTION}

In the 1990s, with more attentions that the people paid to sports, some researchers began to study the relationship between core strength and sports, and finally the core strength was applied to competitive sports, which greatly improved athlete's athletic performances. Since then, the core strength has become an indispensable part for the competitive sports training. Although the core strength has been widely regarded, yet the research on it in various sports is still kept at a low level, in particular, few conduct research on the badminton, a common sport for the people.

\section{DEFINITION AND CHARACTERISTICS OF THE CORE STRENGTH IN BADMINTON SPORT}

With the frequent application of the core strength in competitive sports, many sports fans and the sports field pay more and more attentions to research on the core strength. The

Topic Funds: Research topic of key theory and realistic question in Shaaxi Social Science Field in 2016, Topic Code: $2016 Z 083$

Level: Provincial and ministerial, Topic Name: Research on the Inheritance and Development of Sports Culture Industry in Shaanxi Province under the Background of New Silk Road. researches on the definition and characteristics of the core strength are premises for its application in the competitive sports.

\section{A. Definition}

The core strength mainly refers to the strength of muscles groups on both sides of the trunk of the human body, for the muscles groups on both side of the trunk are small muscle masses supporting the movement, which may control the balance and strength of players during movement. In short, the core strength is a strength generated by core parts supporting the body during movement.

\section{B. Characteristics}

With the gradual expansion of the core strength application, the characteristics of the core strength have become an important part in it. Analyzing from the performance of the core strength in competitive sports, we can draw a conclusion that the core strength generally has the following characteristics:

1) Centered on the core parts of the body: Core parts of the body are the places where main strengths of the body is gathered, which can effectively control the upper and lower limbs and the direction of center of gravity during movement. In contrast to traditional strength training, the core strength works through the core parts of the body, which control the roles of the muscles and nerves in the power transmission so as to coordinate the relationship between strengths.

2) Making players ready for movement: The core strength area is a central area of the body, which works to dominate and regulate the movement, take the badminton sport as an example, the main parts of the strength are the lower limbs and arms during movement, and the time control for the strength of core muscles is earlier than that of the lower limbs and arms. Therefore, during the movement, the core strength can perceive the needs of the body in advance so as to make the player ready for movement in advance ${ }^{[1]}$.

\section{CHARACTERISTICS OF BADMINTON SPORT AND ENERGy METABOLISM DURING MOVEMENT}

Compared with other sports, the badminton is a relatively lightweight sport, popular in the mass, and it has been rapidly popularized throughout the world. The research on the 
badminton sport can be conducted from its characteristics and energy metabolism during the movement.

\section{A. Characteristics}

Characteristics of the badminton during movement mainly include,

1) Aiming at attack during movement: Differing with the movement of basketball or football, the badminton is mainly aiming at attack, after receiving the shuttle from the opposite, the player will increase the intensity of the attack and attack instantly. The attack is one of the main characteristics of badminton, and a fundamental way to score.

2) Quick in attack: The badminton player can control the speed of shuttle through the power generation, but during a badminton competition, it shall be kept quick, and the quick attack can reduce the time of the opponent for observation and thinking, which will cause psychological tension and fear of the opponent so as to disperse the attention of his or her own during the game and achieve the victory of attack

3) Flexible and diverse during movement: The badminton sport has high requirements to the flexibility of the body, for it requires the participation of all parts of the body in the movement, and it is a movement of all parts. Therefore, during the movement, the players should adopt flexible ways of attack according to the opponent's habits and analysis in situ, in order to cause the adverse counterattack of the opponent and get scores.

4) Steady in movement: The badminton sport is to activate the movement of all parts, therefore, during the movement, it focuses on the steadiness whether in the body or the way of $\operatorname{attack}^{[2]}$, namely, during the movement, it requires to balance the body and no imbalance because of receiving it, of which the opponent can take advantage; in addition, during the attack, the player should have a stable technique and consider the shuttle distance, time to receive and attach direction.

\section{B. Characteristics of Energy Metabolism and Sport Loads During the Movement}

Though the badminton sport is lightweight while it is also of typical high intensity, especially during the game, the shuttle goes quickly, thus the player will be in a movement of high speed and high intensity. In this case, the energy metabolism within the body will be in a big change. First of all, with the increase in the movement intensity, the lactic acid content in the body will increase, which will be decomposed into other substances for energy release before seconds of movement and provide conditions for movement, finally through the continued movement, the body will generate new blood lactate for energy supply. Therefore, according to the characteristics of badminton sport, the core strength shall be fully used for training of badminton sport and improve the physical functions of players.

Sport load, an essential element necessary for each movement, consists of load and load strength, which directly affects the effect of the badminton sport and training. During the movement, the difference of the load that the badminton player can bear will generate different functional responses. If the load exceeds the load intensity, the function will be unable to continue the game. So the player shall make full use of the body functions of their own in the game to adjust the load, reduce the consumption of energy to enhance the utilization of physical functions.

\section{TRAining OF THE CORE STRENGTH IN BADMINTON SPORT}

In recent years, with the rising of competitive sports and the core strength proposed, the research on the relationship between core strength and competitive sports has become one of the important issues for researchers, and the training of the core strength of players has also become a training way widely adopted in the sports field. As a nationwide fitness way, how to combine the badminton with the core strength for training will play an active role in the better development of the badminton sport.

\section{A. Significant Roles of the Core Strength Training the Badminton Sport}

Researches on the important roles of the core strength in badminton sport will help to strengthen the understanding of the people to the significance of core strength and help to carry out special training on the core strength for the badminton sport. In accordance with the analysis of the core strength in the badminton sport to the body, energy and safety of players, the roles of the core strength for the badminton sport mainly include the following:

1) Help players to control the body balance: The mass of a shuttle is relatively light; besides, it has a lower request for actions, so it belongs to a lightweight movement. However, the badminton sport emphasizes the body balance, for only the body is kept balanced, and the player may guarantee coherence at feet so as to enhance the quality of the shuttle movement. Besides, during the attack smash, the player needs to control the body balance. All of these are achieved only with the core strength well control.

But as far as the badminton sport, perhaps many think that that the badminton sport only needs the movement of arm muscles, whose technical actions are done by arms. Yet seen from a scientific point of view, the badminton sport can be achieved only with mutual coordination of various parts of the body. In other words, during the badminton sport, the completion of each action requires the control of the core strength.

2) Maintain the balance of energy and improve skills of players: Due to the visual phenomenon, most think that badminton sport is nothing more than the movement of the lower limbs, which help players locate the shuttle for attack, and arms are used to control shuttle attack direction and intensity, so the energy movement during the badminton sport, is also limited to the work of arms.

Actually during the badminton sport, both each movement and energy conversion of the player are controlled by the core strength, which guarantees the orderly movement of energies of the body, and reduces the joint load and enhance the cooperation of the body during movement. 
For example, during the badminton movement, the players mainly get scores through attacks, so in order to successfully get scores through attacks, they have to gather the power to the arm, then through swing the bat with the power transferred to the shuttle. This transfer of the power requires that the core strength should control the whole body for movement and concentration. Thus, the core strength has a good effect in maintaining the balance of energy and improving the skills of the players.

3) Guarantee the safety of the players during movement and prevent injures: In recent years, with the increased need for the living level of the people, fitness has gradually become a fashionable topic, and the badminton sport, as a nationwide sport, are popular in many sports fans. However the overload exercises are inevitably causing some body injuries as joint damages and spinal injury and so on ${ }^{[3]}$.

Yet researches by US experts have shown that the players with better core strength can not only fully maintain the body balance during the movement, owing stronger skills, but also have their muscles quickly restored till relaxed in a short time, which reduce the energy consumption and exercise load so as to guarantee the safety of the players during movement and reduce the risks of injury. In addition, the players with better core strength can keep correct exercise postures during hot movement and lower the energy loss and injuries due to wrong actions.

\section{B. Analysis of the Core Strength Training}

In order to popularize and spread the core strength training in a large scope, first of all, it is necessary to make all be aware of what the core strength training is, the position of the core strength training during the badminton sport, the significance of the core strength as well as training methods of the core strength during the badminton sports. As such, can the core strength training be proposed to the training schedule for the badminton sport.

1) Definition of the core strength training: Facing a sport, the players need to participate in lots of training, such as physical training, skill training and so on. The fundamental purpose of the core strength training is to coordinate the energy balance in the bodies of the players and reduce the load of main joints. Therefore, the core strength training is also a physical training, yet it is much more targeted than general physical training. For the core strength training is to train the physical fitness of the players through coordinating energies in the body.

The core strength training is to train the energies of core parts of the body through the training equipment and methods applicable for the core strength so as to improve the function of the core parts of the body, coordinate the energy balance for movement and stabilize the center of gravity during the badminton sport.

2) Position of the core strength during the badminton sport: The core strength of the badminton players refers not to the strength to control the direction and speed of the shuttle during the movement but the strength and role generated at the core parts of the body during the movement. It is embodied that through the energy transfer, the core parts can keep the body balanced, coordinate the parts of the body and participate in the energy transmission.

The core strength, equivalent to a control panel, will be the first to receive signals and convey to other parts of the body through neural regulation, and control the energy transmission. The essence of its existence is to create a "supporting point" for other parts of the body, based on which, other parts in the movement will achieve the energy transmission.

3) Training methods for the core strength during the badminton sport: In traditional training on the physical training, it is generally done on the surface muscle groups, yet the training on the surface will do bad to the energy coordination in the body of the players during the movement. The core strength training is to train the muscle groups of core parts to achieve the energy flow in the body, which is a training invisible.

Yet it's known that any movement will need the participation of a lot of muscle groups for the completion of exercises, and there are few sports only through single muscle groups, so any movement will need the cooperation of muscle groups of all parts of the body during the movement. Therefore, the fundamental purpose of the core strength is to deploy the muscle groups to enable them to coordinate in the exercise, and enable the players to exert skills during the movement. Methods for the core strength training mainly include:

a) Bare-handed exercise method: Bare-handed exercise method is the most simple and practical method for the core strength training, during the exercise, a movement with some core strength used to fully exert the role of the core strength of the body, and the repeated training will exert the core strength during the badminton sport. Currently main means for the bare-handed exercise method include "get down on one knee with arched back supporting, superman, and curlup, etc.

The training methods have a common characteristic namely it needs the participation of all parts of the body to achieve the exercise, and it is a fully-activated training, which can train the movements of muscles at core areas of the body, fully stimulate the concentration and conversion of strength at the core areas, and make the core strength exert its role during the badminton sport.

b) Simple mechanical exercise: During the core strength training, the bar-handed exercise can only awaken the initiative of the core areas of the body, but in order to make full use of the core strength in a badminton competition, the bare-handed exercise is inadequate, and simple mechanical exercise shall be used for the players. At present, main equipment for the core strength training include Swiss ball, balance board and balance ball and so on. Seen only from the simple equipment, we can find that that these are mainly used for the balance exercise of the players.

The exercises enable the muscle groups in a deep level of the body to participate in maintaining the stability of the body, and exert the core areas of the body and coordinate with other deeper muscle groups to participate in the control of the body balance. For example, the "lying with leg raised and going 
around the ball" in the core strength training enables feet of the player to stand on the balance ball, and the waist muscles are used to drive the foot to make right-left movement, but during the movement, the body should shall be kept stable with the feet on the balance ball. This exercise with simple facilities is a way for the core strength training of the badminton players, for with the body balanced, it could promote the muscle groups of the body and exert the coordination of the core strength between muscle groups.

c) Complex equipment exercise: The exercise is a final stage for the core strength training of the players, generally those who participate in the training have the ability to apply the core strength, for the complex exercise method is suitable for the players who have reached high or medium level in badminton. And it requires the players to use the core strength to balance the body in order to start the next exercises.

The training through the complex equipment is specified as follows: According to the core strength of the trainee, arrange the player to stand on the balance ball with one foot or more, after the player is kept balanced, small barbells will be put on the hands of the player by the coach, to carry out the "squat and stand up" exercise. This training is to adjust the body and keep it balanced, during the exercise, the neutral mechanism will generate adaptable mechanism continuously due to the control of core areas so as to keep the body balanced.

The methods of training for the core strength are practiced from simple to complex, for the core strength training is not a simple process, it requires the constant feeling and adaptation of the body so as to enable the core strength to actively participate in the movement and help the players maintain the body balance and promote badminton skills learning and strengthening.

\section{CONCLUSION}

Seen from the roles of the core strength during the badminton sport, the core strength may balance the body of the player during the movement, and do good to the development of the skills. During the core strength training of the players, the training standards and training methods specified should be combined with the characteristics of the badminton sport and make the actions in training accord with the badminton requirements. In addition, before training, careful analysis shall be conducted on the physical performance of the players, and establish the training plans of core strength according to the physical conditions of their own and do all with purposes..

\section{REFERENCES}

[1] Tang Guoyu, Application of Core Strength Training in Badminton Sport [J]. Shanxi Youth, 2013 (11)

[2] Jia Jingna, Guo Yifeng, Discussion the Core Strength Training for Badminton [J] Jintian, 2012(9)

[3] Xing Zhinan, Important Role of Core Strength Training in Badminton Exercise [J]. Heilongjiang Science and Technology Information, 2014(10)

[4] Zhang Lei, Zhang Xinran, Pan Fan, Discussion and Research on Core Strength Training in the Badminton Exercise [J]. Contemporary Sports Technology, 2013(12). 